\title{
Possibility of Using DNA Chip Technology for Diagnosis of Human Papillomavirus
}

\author{
Cui-hua Liu, Wen-li Ma*, Rong Shi, Yang-qian Ou, Bao Zhang and Wen-ling Zheng** \\ Institute of Molecular Biology, First Uniform Medical University, Guangzhou 510515, China \\ Institute of Molecular Oncology, Guangzhou General Hospital of Guangzhou Command, Guangzhou 510010, China
}

Received 10 December 2002, Accepted 14 February 2003

\begin{abstract}
To explore the application of DNA chip technology for the detection and typing of Human Papillomavirus (HPV), the HPV6, 11, 16 and 18 gene fragments were isolated and printed onto aminosilane-coated glass slides by a PixSys 5500 microarrayer as probes to prepare the HPV gene chips. HPV samples, after being labeled with fluorescent dye by restriction display PCR (RD-PCR) technology, were hybridized with the microarray, which was followed by scanning and analysis. The experimental condition for preparing the HPV gene chips was investigated, and the possibility of HPV genotyping using gene chips was discussed. The technique that was established in this study for preparing HPV gene chips is practical. The results of the present study demonstrated the versatility and inspiring prospect of using this technology to detect and genotype HPV.
\end{abstract}

Keywords: DNA chip technology, Fluorescence labeling, Human papillomavirus (HPV), Random-priming, Restriction display PCR (RD-PCR)

\section{Introduction}

HPVs frequently infect humans. They are classified into categories of low-risk types responsible for the most common sexually-transmitted viral infections and high-risk types which are crucial etiological factors for cervical cancer development (McFadden and Schmann, 2001). It is extremely important to

\footnotetext{
*To whom correspondence should be addressed.

Tel: 020-85148210; Fax: 020-85147755

E-mail: wenli@fimmu.com or wenling@fimmu.com
}

This work was supported by research grants from the National Natural Sciences Foundation of China (No.39880032) as well as the Key Science and Technology Projects of Guangzhou (No.99-Z-022-01). detect and genotype HPVs at an early stage of the infection so as to direct clinical treatment and reduce the incidence of HPV-related carcinomas, especially cervical cancer (Bosch and de Sanjose, 2002). The traditional method for HPV detection, such as morphological and immunological methods, showed low specificity and sensitivity. Currently, the most frequently used methods for HPV detection are molecular biological methods, including nucleic acid hybridization-based and PCR-based methods. But, these methods need improvement, especially in regard to the falsepositive incidence with PCR methods. In this study, we report the application of DNA chip technology for the detection of HPV. We initiated some pilot studies in the preparation of HPV gene probes, manufacture of HPV gene chips, fluorescence labeling, hybridization optimization, etc. We also explored the possibility of using HPV gene chips in genotyping of HPV subtypes using HPV6, 11,16 and 18 as models.

\section{Materials and methods}

\section{Materials and instruments}

HPV plasmids: For this study, 4 types of HPVs (HPV6, 11, 16 and 18) were selected. HPV plasmids including the whole HPV genome were provided by Dr. de Villier from Deutsches Krebsforschungszentrum (Heidelberg, Germany).

Reagents: Klenow Fragment, dNTP, Random Primer (6 mer), Sau3A I, T4 DNA ligase, and E. coli DNA polymerase I were obtained from TaKaRa (Otsu, Japan); 3S PCR purification kit was purchased from BBST (Shanghai, P.R. China); Dimethyl sulfoxide (DMSO) was bought from Sangon (Shanghai, P. R. China); FluoroLink Cy3-dUTP (Cy3), FluoroLink Cy5-dUTP (Cy5) were provided by Amersham Pharmacia (Piscataway, USA).

Instruments: PixSys 5500 gene chip printing machine was purchased from Cartesian Technologies (Irvine, USA); GS Gene Linker Ultraviolet chamber was from BioRad (Hercules, USA); ScanArray Lite was provided by GSI Lumoncis (Billerica, USA); CMT-GAPS $^{\mathrm{TM}}$ coated slides and Corning CMT-Hybridization ${ }^{\mathrm{TM}}$ 
chambers were ordered from Corning Microarray Technology (Acton, USA)

Probe preparation by RD-PCR methods After isolating the HPV genomes from the plasmids, RD-PCR was performed according to the references (Ma W et al., 1998; Zheng W et al., 1998; Ma W et al., 2000). Double-stranded HPV DNAs were digested with Sau3A1, then T4 DNA ligase was used to link the gene fragments with universal adapters. PCR primers were designed to match the universal adapters. Reactions were performed in a 9700 thermocycler with an initial denaturation of $5 \mathrm{~min}$ at $94^{\circ} \mathrm{C}$, followed by 35 cycles of $30 \mathrm{~s}$ at $94^{\circ} \mathrm{C}, 30 \mathrm{~s}$ at $60^{\circ} \mathrm{C}$, $1 \mathrm{~min}$ at $72^{\circ} \mathrm{C}$, and a final extension of $7 \mathrm{~min}$ at $72^{\circ} \mathrm{C}$. Then the PCR product was cloned to $\mathrm{T}$ vectors and sequenced. The target fragments were amplified and purified with isopropyl alcohol and used for microarray probes.

Probe preparation by designing primers to amplify the specific HPV gene fragments PCR primers: General primers were selected from the highly conservative L1 gene (Monos M et al., 1990); Type specific primers were selected from the specific sequence of the E6 gene, as designed by Pao (Pao et al., 1990); We designed other primers that span other parts of the HPV genomes (primers sequences not shown). All of the primers were synthesized by Bioasia Biotechnology (Shanghai, China). The PCR reactions were performed as follows: $94^{\circ} \mathrm{C}$ for $5 \mathrm{~min} ; 94^{\circ} \mathrm{C}$ for $30 \mathrm{~s} ; 60^{\circ} \mathrm{C}$ for $30 \mathrm{~s} ; 72^{\circ} \mathrm{C}$ for $1 \mathrm{~min} ; 35$ cycles; finally, $72^{\circ} \mathrm{C}$ for $8 \mathrm{~min}$.

Microarray preparation and slide processing The DNA probes were resuspended to a maximum of $250-300 \mathrm{ng} / \mu \mathrm{l}$ in $50 \%$ DMSO and transferred to a 384-well plate. The slides were then printed according to the manufacturer's protocol. After the printing process was completed, the immobilization of DNA was done by ultraviolet irradiation using a Ultraviolet Cross-linker. This was followed by baking the array at $80^{\circ} \mathrm{C}$ for $2 \mathrm{~h}$, then the slides were stored in the slide container at room temperature for immediate or later use.

Sample labeling The samples were labeled with $\mathrm{Cy} 3$ or Cy5 using RD-PCR technology. First, the HPV plasmids were digested with Sau $3 A I$, the gene fragments were linked with universal adapters, then the RD-PCR was performed using Cy3 or Cy5 labeled universal primers. The PCR reactions that contained $5 \mu \mathrm{l}$ digested gene fragments, $2 \mu \mathrm{l} \mathrm{Cy} 3$ or $\mathrm{Cy} 5$ labeled universal primers, $25 \mu \mathrm{l} 2 \times$ Premix, $18 \mu \mathrm{l}_{2} \mathrm{O}$, and the PCR amplifications were performed as follows: initial annealing at $95^{\circ} \mathrm{C}$ for $5 \mathrm{~min}$, followed by 35 cycles of denaturation at $95^{\circ} \mathrm{C}$ for $30 \mathrm{~s}$, annealing at
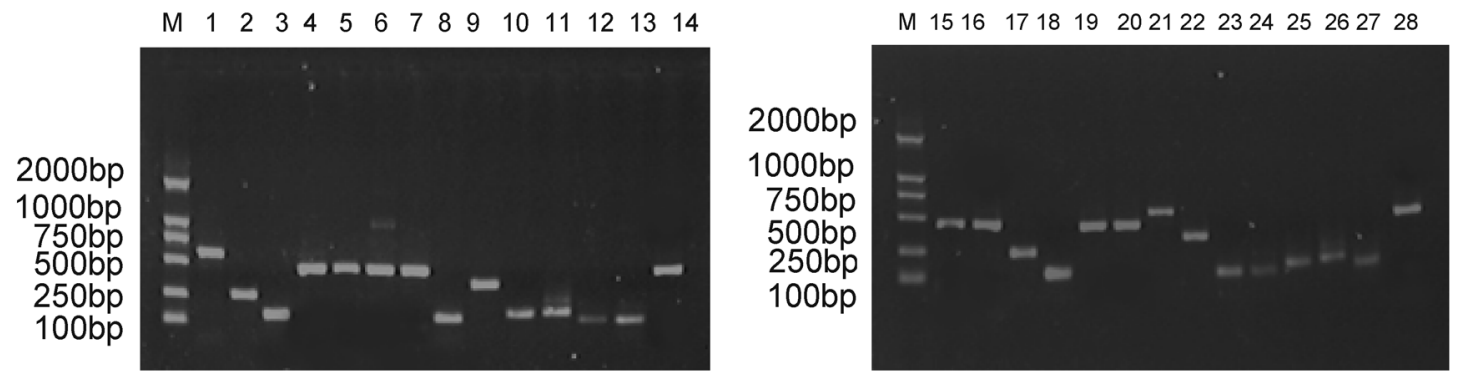

Fig. 1. Purified HPV gene fragments for gene chip preparation. M, DNA marker DL2000; Lanes 1-28, HPV gene fragments. $60^{\circ} \mathrm{C}$ for $30 \mathrm{~s}$, extension at $72^{\circ} \mathrm{C}$ for $1 \mathrm{~min}$, and a final extension at $72^{\circ} \mathrm{C}$ for $5 \mathrm{~min}$. The reaction product was purified and dissolved in $25 \mu \mathrm{l} \mathrm{H}_{2} \mathrm{O}$.

Slide pre-hybridization The slide was incubated in $25 \%$ formamide, $5 \times \mathrm{SSC}, 0.1 \%$ sodium dodecyl sulfate (SDS) in a Coplin jar for $45 \mathrm{~min}$ at $42^{\circ} \mathrm{C}$. It was then rinsed under distilled water 5 times and immersed in isopropyl alcohol for one second, then dried in the air.

Hybridization The Cy3 or Cy5 labeled samples were mixed with an equivalent $2 \times$ hybridization buffer $(50 \%$ formamide, $10 \times \mathrm{SSC}$, $0.2 \% \mathrm{SDS}$ ) and heated at $95^{\circ} \mathrm{C}$ for $5 \mathrm{~min}$. This was followed by centrifugation at 14,000 rpm for 2 min to cool down. The slide was placed in a Corning CMT-Hybridization ${ }^{\mathrm{TM}}$ chamber, then $6 \mu \mathrm{l}$ of the sample solution was pipetted onto the slide surface. Next, a silicon pre-treated coverslip was placed on top of the slide and $10 \mu \mathrm{l}$ water was added into the two holes at both ends of the hybridization chamber to maintain the humidity, after that, the hybridization chamber was submerged in a $42^{\circ} \mathrm{C}$ waterbath for 3-16 h.

Post-hybridization washes A hybridization chamber was dissembled right side up. The coverslip was removed by immersing the slide in $2 \times \mathrm{SSC} / 0.1 \%$ SDS at $42^{\circ} \mathrm{C}$ until the coverslip moved freely from the slide. The slide was placed in $2 \times \mathrm{SSC} / 0.1 \%$ SDS for $5 \mathrm{~min}$ at $42^{\circ} \mathrm{C}$, then was placed in $0.1 \times \mathrm{SSC} / 0.1 \%$ SDS for $5 \mathrm{~min}$ at room temperature. It was then removed into $0.1 \times \mathrm{SSC}$ for $1 \mathrm{~min}$ at room temperature followed by 4 repeats, and the slide was rinsed with running $\mathrm{H}_{2} \mathrm{O}$ and ethanol (100\%) sequentially and dried in the air.

Scanning and analysis The array was scanned with a ScanArray Lite scanner. The results were analyzed using QuantArray array analysis software.

\section{Results}

Probe preparation Twenty-eight of the HPV gene fragments were selected and prepared for HPV gene probes. One $\mu l$ of each was used to perform $1.5 \%$ agarose electrophoresis (Fig. 1). Almost all of them were single fragments and could be used as probes for chip manufacture.

Specificity and reproducibility of hybridization As shown in Fig. 2, the fluorescence-labeled HPV gene 


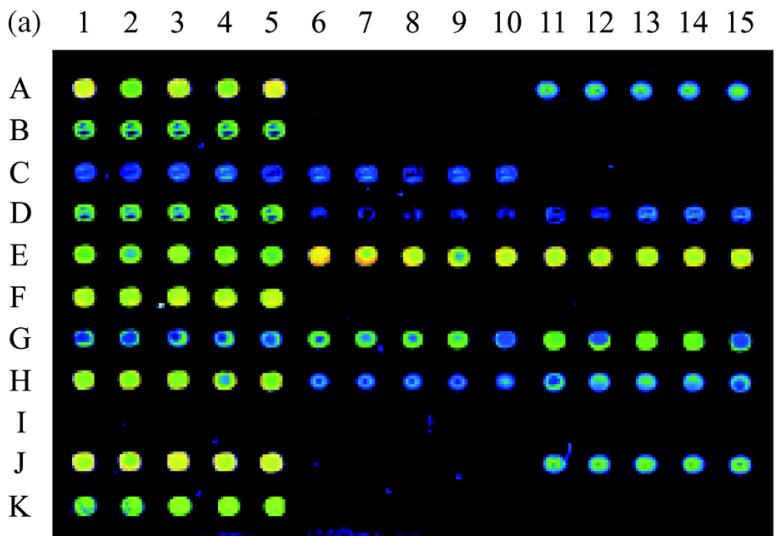

(c)

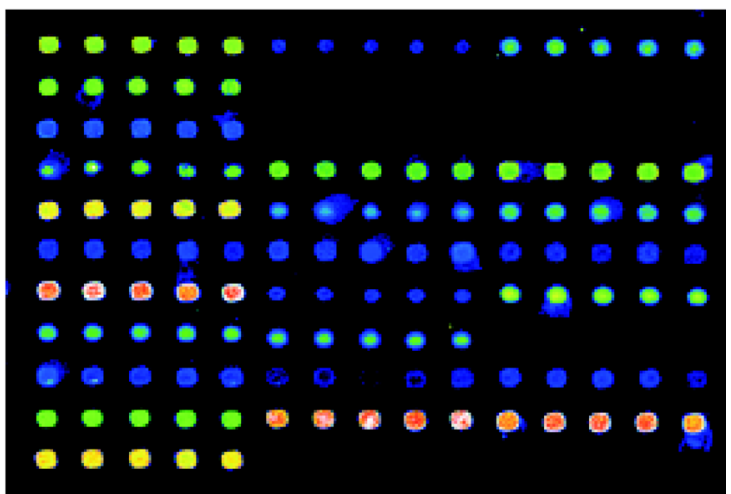

(b)

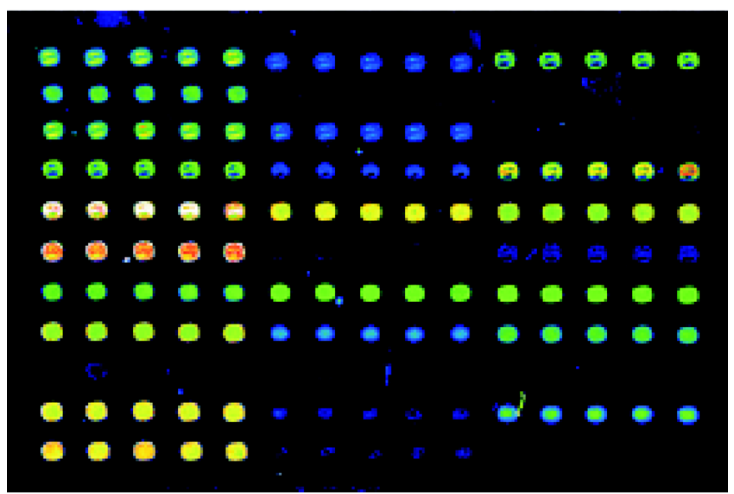

(d)

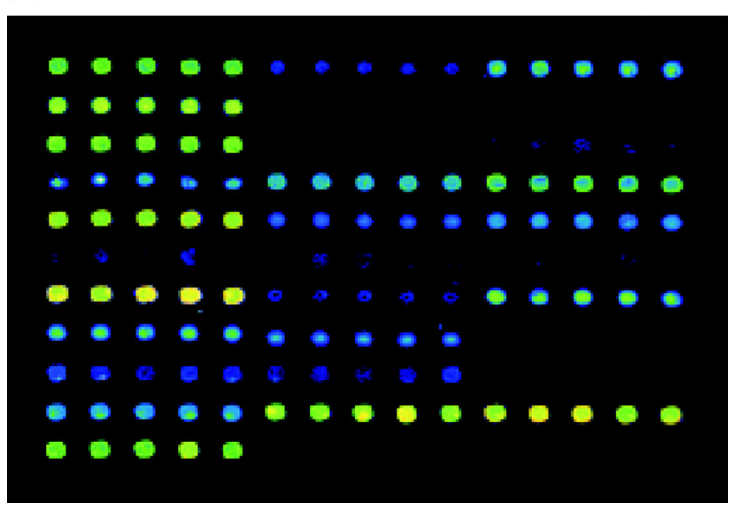

Fig. 2. The array design: The distance between spots was $300 \mu \mathrm{m}$. The total area of the array was $3.3 \times 4.5 \mathrm{~mm}^{2}(15 \times 11)$. Each probe was printed for 5 spots and there were 3 probes per line (for example: A1-A5 for probe 1; A6-A10 for probe 2; A11-A15 for probe 3; B1-B5 for probe 4; B6-B10 for probe 5; etc.). C11-C15 (probe 9), Negative controls (HIV); I11-I15 (probe 27), Negative controls (HCV); B6-B15 (probes 5 and 6) and K11-K15 (probe 33), Blank controls (50\% DMSO); all the other spot: HPV gene fragments. The color ranging from blue to red that followed the change of spectrum represents the gradual fluorescent intensity increment. (a) Hybridization of HPV gene chip with HPV 6, (b) with HPV 11, (c) with HPV 16, (d) with HPV 18.

fragments hybridized specifically with most of the corresponding spots and produced positive signals, while all the negative controls appeared to be negative in chip analysis. This proved high specificity of the array. To demonstrate reproducibility of the array, each probe was printed for 5 spots, and each sample was repeated for 6 times of hybridization. From Fig. 2, we can see that the hybridization signals of every 5 spots of the same probe were consistent. There was a satisfactory consistency among the replicate tests, which demonstrated the high reproducibility of this technical approach.

HPV typing results by gene chip technology To explore the possibility of HPV subtype identification using gene chip technology, we performed hybridization by separately using Cy3-labeled HPV 6, 11, 16, 18 plasmid samples. From the results (Fig. 2), we see that the four different HPV subtypes showed a specific hybridization pattern. There was a similar signal pattern at some spots, especially between the two lowrisk subtypes (HPV 6 and 11). There was at the same time a similar signal pattern between the two high-risk subtypes
(HPV 16 and 18). Nevertheless, distinct differences existed between the low-risk subtypes and their high-risk counterparts.

Sequencing results of the probes To obtain further information of the probes that were printed on the chip, we sequenced and blasted the probes except the controls and those HPV gene fragments that showed weak signals in all the HPV types including probes 2, 8, 17, 18, 25, 26, and 32, because they were less significant in genotyping. The sequencing and analysis results are shown in Table 1.

\section{Discussion}

Optimization of the probe preparation, fluorescence labeling, chip preparation, and hybridization We applied RD-PCR technology, which was invented by our laboratory to collect gene fragments with homogeneous length. We discovered that detecting the same molecular targets with these gene probes can enhance the signal-to-noise ratio and 
Table 1. Information of the sequenced and analyzed probes on the DNA chip

\begin{tabular}{|c|c|c|c|c|}
\hline Probe No. & GenBank No. & Location in genome & Seq. No. (bp) & Length (bp) \\
\hline 1 & X05015.1 & L1 & $6558-7012$ & 455 \\
\hline 3 & X00203.1 & E6 & $140-402$ & 263 \\
\hline 4 & X00203.1 & L1 & $7229-7558$ & 130 \\
\hline 7 & M14119. & L1 & $6707-7155$ & 449 \\
\hline 10 & X00203.11 & L2 & $4546-4726$ & 181 \\
\hline 11 & AF125673.1 & E6 & $50-626$ & 577 \\
\hline 12 & M14119.1 & Poly A signal & $7452-7578$ & 127 \\
\hline 13 & M14119.1 & L1 & $6609-7076$ & 468 \\
\hline 14 & M14119.1 & L2 & $4537-4717$ & 181 \\
\hline 15 & M14119.1 & $\mathrm{E} 4, \mathrm{E} 5$ & $2759-3492$ & 734 \\
\hline 16 & M14119.1 & L1 & $5996-6612$ & 617 \\
\hline 19 & AF125673.1 & L1 & $6583-7034$ & 452 \\
\hline 20 & M14119.1 & E6 & $140-283$ & 144 \\
\hline 21 & X05015.1 & E1 & $1379-1555$ & 177 \\
\hline 22 & X05015.1 & E1,E6,E7 & $150-923$ & 774 \\
\hline 23 & AF125673.1 & L1 & $4539-5076$ & 537 \\
\hline 24 & X00203.1 & $\mathrm{L} 1$ & $6722-7170$ & 449 \\
\hline 28 & X05015.1 & E6 & $91-446$ & 356 \\
\hline 29 & AF125673.1 & Poly A signal & $7422-7587$ & 166 \\
\hline 30 & AF125673.1 & E7 & $622-874$ & 253 \\
\hline 31 & X74479 & L1 & $6562-7016$ & 455 \\
\hline
\end{tabular}

drastically decrease the false-positive rate. We also collected some specific HPV gene probes by designing primers in order to amplify the corresponding HPV gene fragments to further validate and improve the specificity of the microarray.

We dissolved the probes with 50\% DMSO instead of the routinely used SSC, because DMSO can denature the doublestranded DNA to single-strand, which facilitates hybridization and prevents the evaporation of the probe solution. Since we used double-stranded probes, then probe concentrations were not too high in case of renaturation within the complementary strands of the double-stranded probes. According to our experiences, using probes with concentrations of 250-300 $\mu \mathrm{g} /$ $\mu \mathrm{l}$ can achieve satisfactory hybridization results.

Currently, $\mathrm{Cy} 3$ or $\mathrm{Cy} 5$ are the most popular fluorescence labeling materials that are used for chip detection. In pathogen detection, only one kind of fluorescence material is required. In our study, we found that the samples that were labeled with Cy5 resulted in a lower signal-to-noise ratio than $\mathrm{Cy} 3$, so we chose Cy3 to decrease the background and enhance the signal intensity. Since the addition of Cy3-dUTP can have inhibition effects on PCR, then the signal intensity is not positively paralleled with the entering rate of Cy3-dUTP (Yu $\mathrm{H}$ et al., 1994). Therefore, using a relatively lower quantity of Cy3dUTP can also result in a higher PCR efficiency and a good signal-to-noise ratio.

In labeling methods, we compared the random-priming method with the RD-PCR method. The results showed that the former had much lower specificity, sensitivity, and reproducibility than the latter (results not shown). This could be due to the following facts: (1) As a PCR technology, the RDPCR method can exponentially amplify the samples so as to detect trace samples while the random priming method does not amplify the samples. (2) The probes that were achieved by the RD-PCR method had a similar length, so it was easier to control the hybridization dynamics. But, as to the random priming method with its high random access, the probes that were collected by this method were heterogeneous in length, which made the hybridization mechanics hard to control.

The quality of arrays that were produced is highly dependent on the substrate. A poor quality-coated glass slide will lead to problems with spot uniformity and morphology, as well as high and varying background fluorescence. Aldehyde group-coated slides produced a high background while labeling with Cy3. This may be explained by absorption of the exciting light for $\mathrm{Cy} 3$ by the slides themselves. Therefore, we chose amine group-coated slides for the following reasons: (1) The amines carry a positive charge at neutral $\mathrm{pH}$, allowing the attachment of native DNA through the formation of ionic bonds with the negatively-charged phosphate backbone. (2) The electrostatic attachment is supplemented by treatment of the printed substrate with ultraviolet light or heat, which induces free radical-based coupling between the thymidine residues on the DNA and carbons on the alkyl amine. In our experiments, we discovered that using the CMT-GAPSTMcoated slides that were produced by Corning Microarray Technology could achieve uniform DNA retention, low backgrounds, and consistent results.

Molecular hybridization is also one of the key steps of the 
DNA chip technology. To achieve high specificity, the appropriate hybridization condition is necessary. Our experimental results showed that using the hybridization solution that consisted of $25 \%$ formamide- $5 \times \mathrm{SSC}$ and placing the microarray at $42^{\circ} \mathrm{C}$ can achieve relatively constant and specific results.

In addition, other factors such as the incomplete blocking of non-specific sites during the pre-hybridization procedure, inadequate washing after hybridization, scratches, and foreign material on the slide surface can also cause deformation of the array, as well as varying background fluorescence.

Application of DNA chips in HPV genotyping The pathology of HPV is closely related to its subtypes. Therefore, detection and genotypeing of HPV at the early infection stage has very important clinical implications. We used the homemade HPV gene chips to perform the detection of four kinds of HPV subtypes including HPV 6, 11, 16, and 18. From Fig. 2, we can see that the four HPV types showed different hybridization patterns. Because the probes on the array included gene fragments from highly constant L1 and E6 genes of the HPV, the four types of HPVs showed similar hybridization signals at the spots of some homogeneous probes. There were distinct differences in the hybridization patterns between the high- and low-risk HPV subtypes. Figure 2 show that D6-D10 (probe 11) and J6-J10 (probe 29) had higher signals in HPV 16 and 18 than in 6 and 11, while H11-H15 (probe 24) and G6G10 (probe 20) had higher signals in HPV 6 and 11 than in 16 and 18. Further comparison of the HPV typing results also showed differences between HPV 6 and 11, and between 16 and 18. A comparison of HPV 6 and 11 follows: C1-C5 (probe 7), D11-D15 (probe 12), E1-E5 (probe 13), F1-F5 (probe 16) had higher signals in 11 than in 6 . A comparison of 16 and 18 , G1-G5 (probe 19) and J6-J15 (probes 29 and 30) had higher signals in 16, while C1-C5 (probe 7) had a higher signal in 18. Therefore, we concluded that HPV genotyping is possible using microarray approaches. A further analysis of the sequences, GC contents, and $\mathrm{T}_{\mathrm{m}}$ value of the probes showed that these probes were 100-800 bp in length, and had a high GC content (38$55 \%)$ and $\mathrm{T}_{\mathrm{m}}$ values $\left(83-91^{\circ} \mathrm{C}\right)$. As the probes on the array include many different gene fragments from both the constant and the specific genome areas of many different HPV subtypes, we can perform the detection and genotyping by combing several signal patterns, so the false-negative and false-positive incidence can be significantly decreased. Furthermore, by using microarray, we can detect and genotype the HPV in one experiment, which simplifies the procedures and improves the detection efficiency. By screening these probes and collecting more useful probes, we can further optimize the probe design of the microarrays so as to detect and genotype more subtypes of HPV.
Although presently most of the applications of DNA chips have been in the field of oncology, human genetic, or for the monitoring of cellular gene expression, etc.; however, a newly developed technology for gene detection and analysis, DNA microarray technology, provides a very powerful tool, which will probably be of great value for the clinical diagnosis of infectious diseases and the monitoring of therapeutics (Vernet, 2002). Our results demonstrated the feasibility and optimistic prospect of the microarray that is combined with RD-PCR technology in pathogen detection and genotyping (Zhang et al., 2002). Further work is needed to collect more useful gene probes of other HPV subtypes, or other kinds pathogens, and to optimize the probe design. This technology also needs to be tested in the context of clinical trial. When coherent results are established, then other than pathogen detection methods could also be explored with this technological platform.

\section{References}

Bosch F. X. and de Sanjose, S. (2002) Human papillomavirus in cervical cancer. Curr. Oncol. Rep. 4, 175-183.

Ma, W., Zheng, W., Cui, D., Song, Y. and Wu, Q. (2000) DNA microarray chips made on surface of ceramic slides. Acta Bio Bioph Sin. 32, 285-289.

Ma, W., Zheng, W. and James, F. B. (1998) Restriction display: a kind of new technology of differential display; in Progress in Biochemistry and Molecular Biology of Army. Briefing, Sun, Z. X. (ed.), p. 113, Beijing, Uniform Medical Science Press, P. R. China.

McFadden, S. E. and Schumann, L. (2001) The role of human papillomavirus in screening for cervical cancer. J. Am. Acad. Nurse Pract. 13, 116-125.

Monos, M. and Ting, Y. (1990) PCR Protocols: A guide to methods and application, pp. 365-367, Academic Press, San Diego, USA.

Pao, C. C., Lin, C. Y., Maa, J. S., Lai, C. H., Wu, S. Y. and Soong, Y. K. (1990) Detection of human papillomaviruses in cervicovaginal cells using polymerase chain reaction. J. Infect. Dis. 161, 113-115.

Vernet, G. (2002) DNA-chip technology and infectious diseases. Virus Res. 82, 65-71.

Yu, H., Chao, J., Patek, D., Mujumdar, R., Mujumdar, S. and Waggoner, A. S. (1994) Cyanine dye dUTP analogs for enzymatic labeling of DNA probes. Nucleic Acids Res. 22, 3226-3232.

Zhang, B., Ma, W., Hu, Z., Shi, R., Song, Y. and Zheng, W. (2002) A method for evaluation of the quality of DNA microarray spots. J. Biochem. Mol. Biol. 35, 532-535.

Zheng, W., Ma, W. and Waes, C. V. (1998). The differential display of poly A polymerase in tumor cells of differential malignancy; in Investigation on Cell Modulation, Ye, X. S. and Shen, B. F. (eds.), Uniform Medical Publishing, Beijing, P. R. China. 73-79. 